\title{
Hyper-Excitability Followed by Functional Quiescence in Neuronal Cells Caused by Insufficient Cellular Energy (ICE): Treatable by Enhancing the Alternative Cellular Energy (ACE) Pathway
}

\author{
W. John Martin \\ Institute of Progressive Medicine, South Pasadena, CA, USA \\ Email: wjohnmartin@ccid.org
}

How to cite this paper: Martin, W.J. (2017) Hyper-Excitability Followed by Functional Quiescence in Neuronal Cells Caused by Insufficient Cellular Energy (ICE): Treatable by Enhancing the Alternative Cellular Energy (ACE) Pathway. World Journal of Neuroscience, 7, 257-266.

https://doi.org/10.4236/wjns.2017.73021

Received: May 16, 2017

Accepted: June 20, 2017

Published: June 23, 2017

Copyright $\odot 2017$ by author and Scientific Research Publishing Inc. This work is licensed under the Creative Commons Attribution-NonCommercial International License (CC BY-NC 4.0). http://creativecommons.org/licenses/by-nc/4.0/

\begin{abstract}
Living organisms derive energy for cellular activities through three primary mechanisms. The first is photosynthesis, which is restricted to plants and certain bacteria. It uses energy in sunlight to combine carbon dioxide with water to form carbohydrates plus oxygen. The second is chemical energy, which is obtainable by all organisms from the cellular metabolism of carbohydrates and other organic molecules. The third mechanism of obtaining cellular energy is the alternative cellular energy (ACE) pathway. The ACE pathway is expressed as an added dynamic (kinetic) quality of the body's fluids. It results from the absorption of an environmental force termed KELEA (kinetic energy limiting electrostatic attraction). The fundamental role of KELEA is presumably to prevent the fusion and annihilation of electrostatically attracted opposing electrical charges. KELEA can loosen the hydrogen bonding between fluid molecules. KELEA benefits living organisms in part by enabling more efficient biochemical reactions. Cells require a minimal amount of energy to remain viable. Additional energy is required to undertake specialized cellular functions. Illnesses result if cells have insufficient cellular energy (ICE) for their specialized functions. Since KELEA is attracted to separated electrical charges, it is presumably attracted to the electrical charges comprising the membrane potential of cells. It is proposed that the depolarization of neuronal cells leads to the partial release of KELEA for use by the depolarized cell and as a contribution to the overall activation of the body's fluids. Many brain illnesses currently attributed to cellular neurodegeneration are explainable as neuronal cells' adaptations to ICE. The adaptations likely comprise initial hyper-excitability to obtain additional KELEA, followed by functional quiescence prior
\end{abstract}


to actual neuronal cell death. Clinical recovery during both the hyper-excitable and hypoactive phases is potentially achievable by enhancing the ACE pathway. Furthermore, among the restored specialized functions of quiescent neuronal cells may be the capacity to again attract KELEA, leading to sustainable recovery. The opportunity exists for extended clinical trials involving the ACE pathway in neurological and psychiatric illnesses.

\section{Keywords}

Alternative Cellular Energy (ACE) Pathway, Insufficiency of Cellular Energy, ICE, KELEA, Kinetic Energy Limiting Electrostatic Attraction, Neurodegeneration, Membrane Potential, Chemical Reactions, Neurology, Psychiatry

\section{Introduction}

It is widely assumed that work performed by living organisms is fueled entirely, either directly or indirectly, from the energy in sunlight via photosynthesis [1]. Specifically, it is known that chlorophyll in plants and in certain bacteria utilizes sunlight to partition hydrogen ions (protons) across lipid membranes [2]. The lipid membranes contain portals allowing for the electrostatic and chemiosmotic induced return of the protons through the membrane. ATP synthase enzymes are located within these portals [3]. The proton motive force enables the ATP synthase enzymes to add a third phosphate from the phosphoryl $\left(\mathrm{PO}_{3}\right)$ molecule to adenosine diphosphate (ADP) [4]. This creates the high-energy molecule, adenosine triphosphate (ATP) [5]. Essentially, the chlorophyll absorbed electromagnetic energy from sunlight is initially converted to the potential electrical energy of separated charges and this energy is subsequently converted to chemical energy [6]. The reconversion of photosynthetically-derived ATP back to ADP provides the rubisco enzymes in plants with chemical energy to link carbon dioxide with water to form glucose and to release oxygen [7]. The linking of carbon dioxide to water is reversible using a different set of enzymes in an oxygen requiring process called oxidative phosphorylation [8]. The oxidation of glucose in plants, as well as animals, occurs in the cells' mitochondria [9] and yields ATP from ADP for numerous cellular activities [10]. These activities include the synthesis of other types of organic molecules, including the many structural and functional components of organisms. The reconversion of ATP to ADP also supplies energy for the activities of most functional molecules. Replenishing ATP from $\mathrm{ADP}$ is achieved through the biochemical conversion into smaller chemical units (metabolism) of organic molecules consumed as food [11]. The metabolism of food also provides heat in warm blooded animals and humans. Daily energy consumption in an average sized human is equivalent to approximately two thousand kilocalories (2000 kcal), also expressed as 2000 Calories [12].

\section{Additional Cellular Energy beyond the Metabolism of Food}

Upon closer examination, the above explanation does not fully account for the 
work performed by humans. For example, most humans maintain a body temperature greater than $20^{\circ} \mathrm{C}$ above the average temperature of the environment. The body temperature returns to that of the environment well within 24 hours of death. A Calorie (kcal) is required to raise the temperature of 1 kilogram $(\mathrm{Kg})$ of water by $1^{\circ} \mathrm{C}$ [13]. Slightly less heat is required to raise the temperature of solid bodily tissues. Discarding the minor variable that the body is not entirely composed of water, a $75 \mathrm{Kg}$ individual effectively requires $20 \times 75$ or 1500 Calories daily to simply maintain the body at $20^{\circ} \mathrm{C}$ above that of the environment. The remaining 500 Calories from a typical 2000 Calorie diet is clearly insufficient to account for the extensive daily work performed by muscles, including the heart; brain; intestines; liver; kidney; and other body organs.

Another difficulty with the assumption that photosynthesis is the evolutionary source of all cellular energy is the energy requirement to originally synthesize chlorophyll, ATP synthase, lipids, etc. A fundamental requirement for life is establishing membranes that allow the separation of electrical charges. Lipids can create a barrier to the passage of water soluble electrolytes; as can membranes composed of simple terpenes. The formation of hydrophobic membranes, which encapsulate molecules with overall negative charges would lead to an electrical charge differential with the electrically neutral outside environment. Separated electrical charges have potential energy, which is directly related to the level of the opposing charges and the distance between the charges [14]. As separated charges move closer to one another, the decreasing potential electrical energy can be converted into other energy forms. Therefore, having regulated openings or passageways in the membrane can theoretically account for the conversion of potential electrical energy into chemical energy. It is essentially the same process by which photosynthesis and oxidative phosphorylation supposedly generate chemical energy. Indeed, some chemists explain all chemical reactions as being solely driven by an overall reduction in the potential electrical energies of the reacting molecules.

\section{Possible Involvement of KELEA in Conversion of Electrical to Chemical Energy}

A premise of the KELEA hypothesis is that separated electrical charges attract KELEA, which is partially released as the electrical charges approach one another [15]-[22]. The presumed release of KELEA is in addition to the energy resulting from the generally understood reduction of potential electrical energy of approaching electrical charges. Oscillating electrical charges are viewed as a net source of additional energy into living organisms, with only some of the energy necessarily being converted to chemical energy. The enzyme rubisco is relatively inefficient in chemically combining carbon dioxide with water in photosynthesis [23]. Yet it can be shown to function in an oscillatory manner [24] [25] [26]. It is intriguing to consider that rubisco and quite possibly other oscillatory enzymes are partially functioning in the delivery of KELEA to plants.

A more defined postulate is that the depolarization process of electrically ac- 
tive neuronal and neurosensory cells is a major source of KELEA for cellular energy [27] [28] [29]. Repetitive depolarization could lead to an increased availability of KELEA, which is transferable to intracellular water, thereby, improving the dynamic activity of the water. Specifically, the heightened KELEA would facilitate energy requiring, intracellular chemical reactions. These reactions could further augment the supply of KELEA by re-establishing cross-membrane electrical gradients through the selective transport of electrolytes across the cell membrane. In other words, it is proposed that the fluctuating electrical activity of the brain and muscles, including the heart, can act as a positive feedback antenna to usefully capture KELEA from the environment [27] [28] [29].

\section{Hyper- and Hypo-Excitability of Neuronal Cells as Responses to ICE}

As explained above, hyper-excitability of neuronal and/or neurosensory cells could reasonably be a compensatory reaction to ICE [27] [28] [29]. This is consistent with illnesses such as pain syndromes with lower pain threshold, paresthesia, photophobia, epilepsy and various other common hyperactive neurological symptoms. Unlike unicellular organisms, repetitive depolarization of neuronal cells in complex systems such as the brain can impose a potential drain on the available energy. This can occur due to the resulting downstream synthesis and release of neurotransmitters and to the need for added synthesis of electrolyte transport channels [29]. Continued ICE is likely, therefore, to eventually lead to a cessation of the specialized functioning of the neuronal and/or neurosensory cells. Essentially, the cells would become functionally quiescent, at least for some time prior to ultimate cell death. This is not to argue that neuronal cell death and brain atrophy are not ultimate features of neurodegenerative disease [30]. Rather, it is proposed that neurodegeneration may be preceded by a prolonged period of functional quiescence of neuronal cells.

\section{ACE Pigments and KELEA Activation of Water}

The major distinction between functional neuronal quiescence and neurodegeneration is that the former is potentially reversible with additional cellular energy. A possible source of added energy is more efficient metabolism of food. Impaired metabolism of food can arise from deficiencies of specific dietary nutrients, restricted delivery of oxygen, intake or continuing presence of toxins, and various genetic disorders. Increasing or adjusting food intake is, however, of little or no clinical benefit in most patients. The more intriguing possibility is clinical recovery from efforts to enhance the ACE pathway [15]-[22]. Indeed, there are indications of the body's own efforts to increase its uptake of KELEA. This is seen, for example, in the formation of KELEA attracting materials, termed ACE pigments. These materials were initially described as self-assembled particles and fibers that suppress the cytopathic effect of cultured stealth adapted viruses [31] [32]. ACE pigments form intracellularly as inclusions, but are then 
excreted into the extracellular fluid. Chemical and other analyses indicate that the particles comprise a wide range of both aliphatic and aromatic complex hydrocarbons, along with other organic molecules. They are commonly complexed with minerals, which vary widely between different particles. There is an abundant production of lipids, including membranes within the tissue culture medium [31] [32].

A remarkable feature of the recovered virus infected cells is the marked disruption of the cells' mitochondria [33] [34]. This suggested that the particles were substituting as an energy source for the cells. Indeed, ACE pigments have energy transducing properties, including being electrostatic, fluorescent and occasionally ferromagnetic. Fluorescence occurs under both ultraviolet (UV) and visible light. The UV fluorescence can be greatly enhanced with certain dyes, including neutral red and acridine orange. ACE pigment particles and fibers also form in the tissues of stealth adapted virus infected patients [33] [34] [35]. Their presence is a defining feature of an illness referred to as Morgellon's disease [36]. ACE pigments also form in response to conventional virus infections, including herpes simplex virus (HSV), herpes zoster virus (HZV) and human papillomavirus (HPV). Moreover, UV light illumination of neutral red dye stained herpes virus induced skin lesions is a readily available means of expediting healing of the lesions [37] [38] [39].

Creating a heightened KELEA energy field able to activate the ACE pathway may explain the therapeutic effect of several types of medical devices, which employ rapid on-and-off electrical switching. Historical examples include the beam ray of Royal Raymond Rife, the multi-wave oscillator of Georges Lakhovsky, the papimi machine of Panos Pappas, and electro-acupuncture. [Reviewed in 15]. Modern day versions of these various devices have been shown to lower the surface tension and increase the volatility of water that is placed within the heightened KELEA field created by the devices. Energy oscillations can also be created using flashing lights with converging light waves [40] and by bifilar electrical coils conducting bidirectional direct current [41]. Water activation can also be achieved by electrolysis in the production of Brown's gas, which includes a form of non-thermal vaporized water molecules [15].

ACE pigments were identified as being dipolar based on their being electrostatic and prone to self-assembly. As expected, they can readily activate water. So too can many other dipolar compounds when added to water. Examples include humic/fulvic acids, zeolites, mineral oxides, herbal materials, etc., [15]-[22]. When chemicals are used, they can be removed by decanting, filtration or repeated dilutions, as in homeopathy. The activated water can be either parenterally administered or consumed as drinking water. KELEA releasing activated fluids can also be therapeutically effective if simply placed in proximity to the patients. Neutral red dye can be added to the fluid and the solution illuminated with UV light. This latter approach has been successfully used in treating children with autism [42] and is effective even if the body is shielded from the fluorescence by a black plastic barrier. 


\section{Brain and Muscle Activities in Support of the ACE Pathway}

It is widely believed that humor, joy, optimism and serenity improve overall health, while stress is detrimental to health. Certain practices have been codified into major movements including religions, Ayurveda practices, Qigong, Reiki, meditation, yoga, etc., [43] [44]. Prior explanations for these effects have included i) possible induced alterations in levels of neurohormones, such as dopamine and endorphins; ii) differing brain wave oscillatory patterns, with preference given to high frequency gamma waves as detected on the electroencephalogram (EEG) and; iii) dominance of the parasympathetic over the sympathetic autonomic nervous system. A more reasonable explanation is that the various beneficial practices are improving the antenna function of the brain in attracting KELEA from the environment. So too might physical exercise with the fluctuating electrical activity of contracting muscles [45] and possibly stressed bone [46]. KELEA can radiate from activated water and it is quite possible that certain spiritual healers can help instill KELEA into those with whom they come into contact.

\section{Conclusion}

The alternative cellular energy (ACE) pathway is defined as a dynamic (kinetic) quality of the body fluids resulting from the attraction of an external force termed KELEA (kinetic energy limiting electrostatic attraction). The ACE pathway is a major source of cellular energy for living organisms, in addition to the cellular energy derived from food metabolism. KELEA is attracted to separated electrical charges and can be partially released as the charges approach one another. This can occur with certain oscillating dipolar molecules and may also accompany certain chemical reactions. It is further proposed that the attraction and release of KELEA occurs during the depolarization of the cell membrane potential of neuronal and neurosensory cells. This can be a source of cellular energy for the neuronal and neurosensory cells as well as for the entire body. An insufficiency of cellular energy (ICE) can lead to an initial hyper-excitability of neuronal and neurosensory cells, followed by functional quiescence or loss of the specialized cellular functions, without necessarily resulting in cell death (neurodegeneration). Neuronal quiescence is potentially reversible using procedures able to enhance the ACE pathway. Moreover, restoration of KELEA attracting capacity of neuronal and neurosensory cells may lead to a sustainable functional recovery of the cells. Clinical validation of the neuronal quiescence hypothesis is provided by anecdotal reports of restored neurological functions in patients receiving Complementary and Alternative Medicine (CAM) therapies. The stage is now set for major controlled clinical studies. A promising approach is to compare the clinical outcomes in neurological impaired patients consuming either regular or KELEA activated drinking water. Clinicians and leaders of patient support groups are encouraged to participate in these studies.

\section{Acknowledgements}

The Institute of Progressive Medicine is a component of MI Hope Inc., a 
non-profit public charity. Valuable clinical input and insights have been received from various Complementary and Alternative Medicine practitioners.

\section{Conflict of Interest}

None.

\section{References}

[1] Raghavendra, A.S. (1998) Photosynthesis. A Comprehensive Treatise. Cambridge University Press, Cambridge.

[2] Pessarakli, M. (2016) Handbook of Photosynthesis. 3rd Edition, CRC Press, Boca Raton, Florida.

[3] Yoshida, M., Muneyuki, E. and Hisabori T. (2001) ATP Synthase-A Marvelous Rotary Engine of the Cell. National Review Molecular Cell Biology, 2, 669-677. https://doi.org/10.1038/35089509

[4] Boyer, P.D. (1997) The ATP Synthase-A Splendid Molecular Machine. Annual Review Biochemistry, 66, 717-749.

https://doi.org/10.1146/annurev.biochem.66.1.717

[5] Mitchell, P. (1961) Coupling of Phosphorylation to Electron and Hydrogen Transfer by a Chemi-Osmotic Type of Mechanism. Nature, 191, 144-148. https://doi.org/10.1038/191144a0

[6] Mitchell, P. (2011) Chemiosmotic Coupling in Oxidative and Photosynthetic Phosphorylation. Biochimica et Biophysica Acta (BBA)-Bioenergetics, 1807, 1507 1538. https://doi.org/10.1016/j.bbabio.2011.09.018

[7] Anderson, I. and Backlund, A. (2008) Structure and Function of Rubisco. Plant Physiology Biochemistry, 46, 275-291. https://doi.org/10.1016/j.plaphy.2008.01.001

[8] Prebble, J.N. (2010) The Discovery of Oxidative Phosphorylation: A Conceptual Off-Shoot from the Study of Glycolysis. Studies in History and Philosophy of Biological and Biomedical Sciences, 41, 253-262. https://doi.org/10.1016/j.shpsc.2010.07.014

[9] Scheffler, I.E. (2011) Mitochondria. 2nd Edition, John Wiley \& Sons, Hoboken, New Jersey.

[10] Blaxter, K.L. (1989) Energy Metabolism in Animals and Man. Cambridge University Press, Cambridge.

[11] Patton, K.T. and Thibodeau, G.A. (2016) Nutrition and Metabolism. In: Patton, K.T., Ed., Anatomy \& Physiology, 9th Edition, Elsevier, Amsterdam.

[12] Bowman, S.A. and Vinyard, B.T. (2004) Fast Food Consumption of U.S. Adults: Impact on Energy and Nutrient Intakes and Overweight Status. The Journal of the American College of Nutrition, 23, 163-168. https://doi.org/10.1080/07315724.2004.10719357

[13] Hargrove, J.L. (2007) Does the History of Food Energy Units Suggest a Solution to "Calorie Confusion"? Nutrition Journal, 6, 44. https://doi.org/10.1186/1475-2891-6-44

[14] Myers, R.L. (2006) The Basics of Physics. Greenwood Press, Santa Barbara, California.

[15] Martin, W.J. (2014) KELEA Activated Water-Enhancing the Alternative Cellular Energy (ACE) Pathway. In: Martin, W.J., Ed., Stealth Adapted Viruses, Alternative Cellular Energy (ACE) \& KELEA Activated Water, Author House, Bloomington, Indiana. 
[16] Martin, W.J. (2015) KELEA: A Natural Energy that Seemingly Reduces Intermolecular Hydrogen Bonding in Water and Other Liquids. Open Journal of Biophysics, 5, 69-79. https://doi.org/10.4236/ojbiphy.2015.53006

[17] Martin, W.J. (2015) Alternative Cellular Energy Pathway Therapy Using KELEA Activated Water. International Journal Complementary \& Alternative Medicine, 2, 00051. https://doi.org/10.15406/ijcam.2015.02.00051

[18] Martin, W.J. (2015) KELEA Activation of Water and Other Fluids for Health, Agriculture and Industry. Journal of Water Resource and Protection, 7, 1331-1344. https://doi.org/10.4236/jwarp.2015.716108

[19] Martin, W.J. (2016) Insufficiency of Cellular Energy (ICE): The Basis for Many Illnesses Potentially Correctable Using KELEA Activated Water. International Journal Complementary \& Alternative Medicine, 4, Article ID: 00106.

[20] Martin, W.J. (2016) Deconstructing Medicine. The Alternative Cellular Energy Pathway. British Journal of Medicine \& Medical Research, 11, 1-6. https://doi.org/10.9734/BJMMR/2016/21484

[21] Martin, W.J. (2015) Alternative Cellular Energy as a Unifying Concept in Complementary Alternative Medicine. International Journal Complementary \& Alternative Medicine, 1, Article ID: 00022. https://doi.org/10.15406/ijcam.2015.01.00022

[22] Martin, W.J. (2016) Cancer as an Insufficiency of Cellular Energy (ICE): Therapeutic Approaches Based on Enhancing the Alternative Cellular Energy (ACE) Pathway. International Journal Complementary \& Alternative Medicine, 3, Article ID: 00074. https://doi.org/10.15406/ijcam.2016.03.00074

[23] Spreitzer, R.J. and Salvucci, M.E. (2002) Rubisco: Structure, Regulatory Interactions, and Possibilities for a Better Enzyme. Annual Review Plant Biology, 53, 449-475. https://doi.org/10.1146/annurev.arplant.53.100301.135233

[24] Teruo, O. (1982) Simple Oscillations in Photosynthesis of Higher Plants. Biochimica et Biophysica Acta (BBA)-Bioenergetics, 681, 103-109. https://doi.org/10.1016/0005-2728(82)90283-3

[25] Laisk, A., Siebke, K., Gerst, U., Eichelmann, H., Oja, V. and Heber, U. (1991) Oscillations in Photosynthesis are Initiated and Supported by Imbalances in the Supply of ATP and NADPH to the Calvin Cycle. Planta, 185, 554-562. https://doi.org/10.1007/BF00202966

[26] Roussel, M.R., Alexander, A.I. and Igamberdiev, A.U. (2007) Oscillations of the Internal CO2 Concentration in Tobacco Leaves Transferred to Low CO2. Journal of Plant Physiology, 164, 1188-1196. https://doi.org/10.1016/j.jplph.2006.08.004

[27] Martin, W.J. (2015) Is the Brain an Activator of the Alternative Cellular Energy (ACE) Pathway? International Journal of Complementary \& Alternative Medicine, 1, 00002. https://doi.org/10.15406/ijcam.2015.01.00002

[28] Martin, W.J. (2016) Insufficiency of Cellular Energy (ICE) in Neurons, From Electrical Hyperactivity to Quiescence. International Journal of Complementary \& Alternative Medicine, 4, Article ID: 00118.

[29] Martin, W.J. (2017) Insufficiency of Cellular Energy (ICE) May Precede Neurodegeneration in Alzheimer's Disease and be Treatable via the Alternative Cellular Energy (ACE) Pathway. Advances in Alzheimer's Disease, 6, 1-12. https://doi.org/10.4236/aad.2017.61001

[30] Sabuncu, M.R., Desikan, R.S., Sepulcre, J., Yeo, B.T., Liu, H., Schmansky, N.J., et al. (2011) The Dynamics of Cortical and Hippocampal Atrophy in Alzheimer Disease. Archives Neurology, 68, 1040-1048. https://doi.org/10.1001/archneurol.2011.167

[31] Martin, W.J. (2003) Stealth Virus Culture Pigments: A Potential Source of Cellular 
Energy. Experimental Molecular Pathology, 74, 210-223.

https://doi.org/10.1016/S0014-4800(03)00037-6

[32] Martin, W.J. (2014) The Alternative Cellular Energy (ACE) Pathway in the Repair of the Cytopathic Effect (CPE) Caused by Stealth Adapted Viruses. In Vitro and In Vivo Evidence Supporting a New Therapeutic Paradigm. In: Stealth Adapted Viruses; Alternative Cellular Energy (ACE) \& KELEA Activated Water, Author House, Bloomington, IN, 31-70.

[33] Martin, W.J. (1996) Severe Stealth Virus Encephalopathy Following Chronic Fatigue Syndrome-Like Illness: Clinical and Histopathological Features. Pathobiology, 64, 1-8. https://doi.org/10.1159/000163999

[34] Martin, W.J. (2003) Complex Intracellular Inclusions in the Brain of a Child with a Stealth Virus Encephalopathy. Experimental Molecular Pathology, 74, 179-209. https://doi.org/10.1016/S0014-4800(03)00038-8

[35] Martin, W.J. (2005) Alternative Cellular Energy Pigments Mistaken for Parasitic Skin Infestations. Experimental Molecular Pathology, 78, 212-214. https://doi.org/10.1016/j.yexmp.2005.01.007

[36] Savely, V.R. and Stricker, R.B. (2007) Morgellons Disease: The Mystery Unfolds. Expert Review Dermatology, 2, 585-591. https://doi.org/10.1586/17469872.2.5.585

[37] Martin, W.J. and Stoneburner, J. (2005) Symptomatic Relief of Herpetic Skin Lesions Utilizing an Energy Based Approach to Healing. Experimental Molecular Pathology, 78, 131-134. https://doi.org/10.1016/j.yexmp.2004.10.007

[38] Martin, W.J. and Stoneburner, J. (2014) Alternative Cellular Energy (ACE) Pathway Activation as the Mode of Action of Neutral Red Dye Phototherapy of Human Viruses. Journal Human Virology \& Retrovirology, 1, Article ID: 00019. https://doi.org/10.15406/jhvrv.2014.01.00019

[39] Martin, W.J. (2014) Neutral Red Dye/Ultraviolet Light Activation of the Alternative Cellular Energy (ACE) Pathway: A Historical Overview and Current Use of Neutral Red Dye Phototherapy of Herpes Simplex Virus Infections. In: Martin, W.J., Ed., Stealth Adapted Viruses, Alternative Cellular Energy (ACE) and KELEA Activated Water, Author House, Indiana.

[40] Martin, W.J. (2015) Interacting Light Paths Attract KELEA (Kinetic Energy Limiting Electrostatic Attraction) and Can Lead to the Activation of Water. Open Journal of Biophysics, 5, 115-121. https://doi.org/10.4236/ojbiphy.2015.54010

[41] Martin, W.J. (2015) Interactive Electric Fields Can Attract KELEA (Kinetic Energy Limiting Electrostatic Attraction) and Can Lead to the Activation of Water. International Journal Complementary \& Alternative Medicine, 1, Article ID: 00034. https://doi.org/10.15406/ijcam.2015.01.00034

[42] Martin, W.J. (2014) Alternative Cellular Energy (ACE) Pathway Activation as Natural Therapy for Autism. In: Martin, W.J., Ed., Stealth Adapted Viruses; Alternative Cellular Energy (ACE) and KELEA Activated Water, Author House, Indiana.

[43] Institute of Medicine (2005) Complementary and Alternative Medicine in the United States. National Academies Press, Washington (DC).

[44] Levin, J. (2009) How Faith Heals: A Theoretical Model. Explore (NY), 5, 77-96. https://doi.org/10.1016/j.explore.2008.12.003

[45] Kuo, I.Y. and Ehrlich, B.E. (2015) Signaling in Muscle Contraction. Cold Spring Harbor Perspectives Biology, 7, Article ID: a006023. https://doi.org/10.1101/cshperspect.a006023

[46] Nakamura, M., Hiratai, R. and Yamashita, K. (2012) Bone Mineral as an Electrical Energy Reservoir. Journal of Biomedical Materials Research, 100, 1368-1374.

https://doi.org/10.1002/jbm.a.34076 


\section{Abbreviations}

ACE: alternative cellular energy, ICE: insufficiency of cellular energy, KELEA: kinetic energy limiting electrostatic attraction, CAM: complementary and alternative medicine, ATP: adenosine triphosphate, ADP: adenosine diphosphate, kcal: kilocalories, Kg: kilogram

Submit or recommend next manuscript to SCIRP and we will provide best service for you:

Accepting pre-submission inquiries through Email, Facebook, LinkedIn, Twitter, etc. A wide selection of journals (inclusive of 9 subjects, more than 200 journals)

Providing 24-hour high-quality service

User-friendly online submission system

Fair and swift peer-review system

Efficient typesetting and proofreading procedure

Display of the result of downloads and visits, as well as the number of cited articles Maximum dissemination of your research work

Submit your manuscript at: http://papersubmission.scirp.org/

Or contactwjns@scirp.org 